Correction

\title{
Correction: Koutsouris et al. Utilization of Global Precipitation Datasets in Data Limited Regions: A Case Study of Kilombero Valley, Tanzania. Atmosphere, 2017, 8, 246
}

\author{
Alexander J. Koutsouris ${ }^{1, *}$, Jan Seibert ${ }^{2}$ (D) and Steve W. Lyon ${ }^{1,3}$ \\ 1 Department of Physical Geography, Stockholm University, 10691 Stockholm, Sweden; \\ steve.lyon@natgeo.su.se \\ 2 Department of Geography, Hydrology and Climate, University of Zurich, 8006 Zurich, Switzerland; \\ jan.seibert@geo.uzh.ch \\ 3 The Nature Conservancy, Delmont, NJ 08314, USA \\ * Correspondence: alexander.koutsouris@natgeo.su.se; Tel.: +46-8-162-000
}

Received: 3 April 2018; Accepted: 3 April 2018; Published: 16 April 2018

The authors would like to correct the published article [1], following the detection of editorial mistakes by the main author, as explained below. Table 3 has been replaced with a new Table to show the streamflow simulation results for Mpanga Catchment. A missing table has been inserted as Table 5, showing the streamflow simulation results for Kiburubutu Catchment. The table previously labeled and referenced as Table 5 should now be considered as Table 6 . All sentences that were referenced to Table 5 refer to the new Table 5, except for the sentence "Simulations based on QM bias corrected GPD products performances were in general worse compared to their non-bias corrected counterparts (Table 5)", which refers to Table 6. Table 3, Table 5, and Table 6 should read: 
Table 3. Performance scores for MC discharge simulations. Performance is shown as $R_{e f f, l o g}, R^{2}, V_{e}$, and $R M S E$ values for each bias correction technique considered.

\begin{tabular}{|c|c|c|c|c|c|c|c|c|c|c|c|c|}
\hline \multicolumn{13}{|c|}{ Mpanga Catchment } \\
\hline GDP & & & $R_{e f f, l o g}$ & $R^{2}$ & $V_{e}$ & RMSE & & & $R_{e f f, l o g}$ & $R^{2}$ & $V_{e}$ & RMSE \\
\hline CFSR & \multirow{10}{*}{ Non- } & \multirow{10}{*}{ bias corrected } & 0.33 & 0.23 & 0.96 & 0.28 & \multirow{10}{*}{ ModB } & \multirow{10}{*}{ bias corrected } & 0.43 & 0.40 & 1.00 & 0.26 \\
\hline ERAi & & & 0.53 & 0.48 & 0.96 & 0.23 & & & 0.53 & 0.50 & 0.96 & 0.23 \\
\hline MERRA & & & 0.38 & 0.44 & 0.94 & 0.29 & & & 0.61 & 0.58 & 0.99 & 0.22 \\
\hline CMORPH & & & 0.41 & 0.32 & 0.95 & 0.27 & & & 0.41 & 0.33 & 0.95 & 0.27 \\
\hline TRMMv7 & & & 0.45 & 0.36 & 1.00 & 0.27 & & & 0.51 & 0.42 & 0.99 & 0.24 \\
\hline Ensemble & & & 0.55 & 0.49 & 0.97 & 0.23 & & & 0.56 & 0.53 & 0.99 & 0.23 \\
\hline Rain gauge & & & 0.12 & 0.14 & 0.94 & 0.36 & & & 0.47 & 0.34 & 0.97 & 0.25 \\
\hline $\mathrm{CRU}^{*}$ & & & 0.27 & 0.17 & 0.98 & 0.30 & & & 0.30 & 0.20 & 0.96 & 0.29 \\
\hline GPCC * & & & 0.29 & 0.23 & 0.99 & 0.30 & & & 0.32 & 0.24 & 0.97 & 0.29 \\
\hline UDEL * & & & 0.35 & 0.25 & 0.99 & 0.28 & & & 0.45 & 0.37 & 0.99 & 0.25 \\
\hline CFSR & \multirow{6}{*}{$\mathrm{QM}$} & \multirow{6}{*}{ bias corrected } & 0.22 & 0.18 & 0.96 & 0.33 & \multirow{6}{*}{$\mathrm{QM}+\mathrm{ModB}$} & \multirow{6}{*}{ bias corrected } & 0.53 & 0.40 & 0.98 & 0.23 \\
\hline ERAi & & & 0.18 & 0.16 & 0.91 & 0.35 & & & 0.54 & 0.41 & 0.98 & 0.23 \\
\hline MERRA & & & 0.05 & 0.08 & 1.00 & 0.33 & & & 0.52 & 0.45 & 0.98 & 0.23 \\
\hline CMORPH & & & 0.35 & 0.22 & 1.00 & 0.28 & & & 0.51 & 0.40 & 0.98 & 0.23 \\
\hline TRMMv7 & & & 0.19 & 0.15 & 0.91 & 0.35 & & & 0.51 & 0.42 & 0.98 & 0.24 \\
\hline Ensemble & & & 0.19 & 0.16 & 0.91 & 0.35 & & & 0.50 & 0.36 & 0.98 & 0.24 \\
\hline GPCC * & \multirow{3}{*}{$\mathrm{DP}$} & \multirow{3}{*}{ bias corrected } & 0.34 & 0.19 & 0.99 & 0.29 & \multirow{3}{*}{$\mathrm{DP}+\mathrm{ModB}$} & \multirow{3}{*}{ bias corrected } & 0.37 & 0.23 & 0.97 & 0.28 \\
\hline CRU * & & & 0.36 & 0.24 & 0.97 & 0.28 & & & 0.38 & 0.27 & 0.95 & 0.28 \\
\hline UDEL * & & & 0.46 & 0.32 & 0.96 & 0.27 & & & 0.52 & 0.44 & 1.00 & 0.24 \\
\hline
\end{tabular}


Table 5. Performance scores for KC discharge simulations. Performance is shown as $R_{e f f, l o g}, R^{2}$, Volume Error (Vol. Err.), and RMSE values for each bias correction technique considered.

\begin{tabular}{|c|c|c|c|c|c|c|c|c|c|c|c|c|}
\hline \multicolumn{13}{|c|}{ Kiburubutu Catchment } \\
\hline GDP & & & $R_{e f f, l o g}$ & $R^{2}$ & Vol. Err. & RMSE & & & $R_{e f f, l o g}$ & $R^{2}$ & Vol. Err. & RMSE \\
\hline CFSR & \multirow{10}{*}{ Non- } & \multirow{10}{*}{ bias corrected } & 0.38 & 0.01 & 0.78 & 0.26 & \multirow{10}{*}{ ModB } & \multirow{10}{*}{ bias corrected } & 0.41 & 0.01 & 0.74 & 0.25 \\
\hline ERAi & & & 0.63 & 0.26 & 0.78 & 0.18 & & & 0.66 & 0.24 & 0.73 & 0.18 \\
\hline MERRA & & & 0.47 & 0.25 & 0.86 & 0.20 & & & 0.68 & 0.24 & 0.72 & 0.18 \\
\hline CMORPH & & & 0.56 & 0.08 & 0.79 & 0.23 & & & 0.62 & 0.10 & 0.68 & 0.20 \\
\hline TRMMv7 & & & 0.55 & 0.07 & 0.87 & 0.23 & & & 0.59 & 0.11 & 0.70 & 0.20 \\
\hline Ensemble & & & 0.63 & 0.19 & 0.80 & 0.19 & & & 0.67 & 0.22 & 0.73 & 0.19 \\
\hline Rain gauge & & & 0.52 & 0.25 & 0.96 & 0.23 & & & 0.59 & 0.21 & 0.78 & 0.20 \\
\hline $\mathrm{CRU}^{*}$ & & & 0.42 & 0.09 & 0.97 & 0.29 & & & 0.57 & 0.12 & 0.70 & 0.21 \\
\hline GPCC * & & & 0.38 & 0.14 & 0.79 & 0.24 & & & 0.59 & 0.14 & 0.69 & 0.21 \\
\hline UDEL * & & & 0.47 & 0.12 & 0.86 & 0.23 & & & 0.64 & 0.16 & 0.71 & 0.19 \\
\hline CFSR & \multirow{6}{*}{$\mathrm{QM}$} & \multirow{6}{*}{ bias corrected } & 0.41 & 0.07 & 0.92 & 0.27 & \multirow{6}{*}{$\mathrm{QM}+\mathrm{ModB}$} & \multirow{6}{*}{ bias corrected } & 0.53 & 0.12 & 0.71 & 0.20 \\
\hline ERAi & & & 0.49 & 0.14 & 0.91 & 0.27 & & & 0.59 & 0.20 & 0.79 & 0.20 \\
\hline MERRA & & & 0.40 & 0.07 & 0.96 & 0.29 & & & 0.53 & 0.12 & 0.75 & 0.21 \\
\hline CMORPH & & & 0.44 & 0.05 & 0.92 & 0.27 & & & 0.51 & 0.08 & 0.74 & 0.22 \\
\hline TRMMv7 & & & 0.45 & 0.04 & 0.98 & 0.29 & & & 0.52 & 0.04 & 0.77 & 0.23 \\
\hline Ensemble & & & 0.48 & 0.07 & 0.91 & 0.29 & & & 0.56 & 0.06 & 0.83 & 0.23 \\
\hline GPCC * & \multirow{3}{*}{$\mathrm{DP}$} & \multirow{3}{*}{ bias corrected } & 0.51 & 0.20 & 0.96 & 0.24 & \multirow{3}{*}{$\mathrm{DP}+\mathrm{ModB}$} & \multirow{3}{*}{ bias corrected } & 0.59 & 0.12 & 0.82 & 0.22 \\
\hline CRU * & & & 0.51 & 0.13 & 0.99 & 0.26 & & & 0.57 & 0.18 & 0.83 & 0.21 \\
\hline UDEL * & & & 0.56 & 0.24 & 0.99 & 0.23 & & & 0.63 & 0.23 & 0.82 & 0.19 \\
\hline
\end{tabular}


Table 6. Performance scores for KC discharge simulations. Performance is shown as $R_{\text {eff, } \log \text { values for }}$ each bias correction technique considered.

\begin{tabular}{|c|c|c|c|c|c|c|c|c|c|c|c|}
\hline \multirow[b]{2}{*}{$\begin{array}{l}\text { Daily } \\
\text { GDPs }\end{array}$} & \multicolumn{6}{|c|}{ Bias corrected performance - KC $\left(\mathbf{R}_{\text {eff,log }}\right)$} & \multicolumn{5}{|c|}{ Change in bias corrected performance - KC ( $\Delta R_{\text {eff,log }}$} \\
\hline & $\begin{array}{l}\text { Non-bias } \\
\text { corrected }\end{array}$ & QM & $\mathrm{DP}$ & ModB & $\begin{array}{c}\text { QM+ } \\
\text { ModB }\end{array}$ & $\begin{array}{c}\text { DP+ } \\
\text { ModB } \\
\end{array}$ & QM & DP & ModB & $\begin{array}{c}\text { QM+ } \\
\text { ModB }\end{array}$ & $\begin{array}{c}\text { DP+ } \\
\text { ModB }\end{array}$ \\
\hline CFSR & 0.38 & 0.41 & - & 0.41 & $\triangle 0.53$ & - & 仓 0.03 & - & 仓 0.03 & 仓 0.15 & - \\
\hline ERA-i & $\triangle 0.63$ & 0.49 & - & $\triangle 0.66$ & $\triangle 0.59$ & - & $\sqrt{-0.15}$ & - & 仓 0.02 & $\sqrt{-0.04}$ & - \\
\hline MERRA & 0.47 & 0.40 & - & $\triangle 0.68$ & $\triangle 0.53$ & - & $\sqrt{-0.07}$ & - & 仓 0.20 & 仓 0.06 & - \\
\hline CMORPH & $\triangle 0.56$ & 0.44 & - & $\triangle 0.62$ & $\triangle 0.51$ & - & $\sqrt{-0.12}$ & - & 仓 0.05 & $\sqrt{-0.06}$ & - \\
\hline TRMMv7 & $\triangle 0.55$ & 0.45 & - & $\triangle 0.59$ & $\triangle 0.52$ & - & $\sqrt{-0.10}$ & - & 仓 0.04 & $\checkmark-0.03$ & - \\
\hline Ensemble & $\triangle 0.63$ & 0.48 & - & $\triangle 0.67$ & $\triangle 0.56$ & - & $\sqrt{-0.15}$ & - & 仓े 0.04 & $\sqrt{-0.07}$ & - \\
\hline Rain gauge & $\triangle 0.52$ & - & - & $\triangle 0.59$ & - & - & - & - & 仓 0.07 & - & - \\
\hline CRU & 0.42 & - & $\triangle 0.51$ & $\triangle 0.57$ & - & $\triangle 0.57$ & - & 仓 0.09 & 仓 0.15 & - & 仓 0.15 \\
\hline GPCC & 0.38 & - & $\triangle 0.51$ & $\triangle 0.59$ & - & $\triangle 0.59$ & - & 仓 0.13 & 仓 0.21 & - & 仓 0.21 \\
\hline UDEL & 0.47 & - & $\triangle 0.56$ & $\triangle 0.64$ & - & $\triangle 0.63$ & - & 仓 0.09 & 仓 0.16 & - & 仓 0.16 \\
\hline
\end{tabular}

$\bigcirc$ Good $\geq 0.70 \triangle$ Fair $<0.7$ and $\geq 0.50 \bigcirc$ Poor $<0.50$

仓 Increase $>0$ Decrease $>0$ No Change $=0$

The authors apologize for any inconvenience this has caused to the readers. The changes do not affect the scientific results of this paper. The manuscript will be updated, and the original version will remain online on the article webpage, with a reference to this Correction.

\section{Reference}

1. Koutsouris, A.J.; Seibert, J.; Lyon, S.W. Utilization of Global Precipitation Datasets in Data Limited Regions: A Case Study of Kilombero Valley, Tanzania. Atmosphere. 2017, 8, 246. [CrossRef] 